\title{
Acknowledgement to Reviewers of Gels in 2016
}

\author{
Gels Editorial Office \\ Published: 11 January 2017 \\ MDPI AG, St. Alban-Anlage 66, 4052 Basel, Switzerland; gels@mdpi.com
}

The editors of Gels would like to express their sincere gratitude to the following reviewers for assessing manuscripts in 2016.

We greatly appreciate the contribution of expert reviewers, which is crucial to the journal's editorial process. We aim to recognize reviewer contributions through several mechanisms, of which the annual publication of reviewer names is one. Reviewers receive a voucher entitling them to a discount on their next MDPI publication and can download a certificate of recognition directly from our submission system. Additionally, reviewers can sign up to the service Publons (https://publons.com) to receive recognition. Of course, in these initiatives we are careful not to compromise reviewer confidentiality. Many reviewers see their work as a voluntary and often unseen part of their role as researchers. We are grateful to the time reviewers donate to our journals and the contribution they make.

If you are interested in becoming a reviewer for Gels, see the link at the bottom of the webpage http://www.mdpi.com/reviewers.

The following reviewed for Gels in 2016:

Abul-Haija, Yousef M.
Ahadian, Samad
Ahearne, Mark
Alvarez-Ramirez, Jose
Andrianov, Alexander K.
Appelhans, Dietmar
Bernards, Matt
Boluk, Yaman
Callahan, Laura Smith
Carmen Sánchez, María Del
Corrias, Anna
Da Silva, Marcelo
Dijkstra, Piet
Dostalek, Jakub
Douglas, Jack F.
Dräger, Gerald
Fontaine, Magali
Winkler, Roland G.
Gohy, Jean-Francois

He, Weidong
Herrera, Raquel P.
Ikeda, Masato
Jain, Gaurav
Jajam, Kailash
Jana, Sadhan
John, Vijay
Jung, Jong Hwa
Kazakov, Sergey
Kharkar, Prathamesh
Kiryukhin, Maxim V.
Lenfant, Gilles
Lupi, Francesca Romana
Marchesan, Silvia
Merino, Sonia
Meyer, Franck
Mignon, Arn
Miyatake, Tomohiro

\author{
Nonappa, Nonappa \\ Okada, Tomohiko \\ Pan, Lijia \\ Paradiso, Vito Michele \\ Russo, Laura \\ Samitier, Josep \\ Shen, Jana \\ Tabata, Makoto \\ Tomasini, Claudia \\ Tonelli, Alan E. \\ Torres, María D. \\ Truong, Vinh \\ Vert, Michel \\ Vogel, Nicolas \\ Wang, Hui-Liang \\ Wang, Liang \\ Weon, Byung Mook \\ Wöll, Dominik
}

(C) 2017 by the authors; licensee MDPI, Basel, Switzerland. This article is an open access article distributed under the terms and conditions of the Creative Commons Attribution (CC-BY) license (http://creativecommons.org/licenses/by/4.0/). 